\title{
Factors Associated with Persistent Smoking Original Factors Associated with Persistent Smoking $\mid$ Article after the Diagnosis of Cardiovascular Disease
}

\section{Hyo-Eun Kim, Yun-Mi Song*, Bo-Kyoung Kim', Yong-Soon Park², Mi-Hyun Kim}

Department of Family Medicine, Samsung Medical Center, Sungkyunkwan University School of Medicine; ${ }^{1}$ Total Healthcare Center, Kangbuk Samsung Hospital, Sungkyunkwan University School of Medicine, Seoul; ${ }^{2}$ Department of Family Medicine, Hallym University Chuncheon Sacred Heart Hospital, Chuncheon, Korea

Background: Although cigarette smoking is a major modifiable risk factor for the occurrence of primary and secondary cardiovascular disease (CVD), not all survivors from CVD attacks can successfully stop smoking. However, little is known about the factors associated with the change in smoking behavior after CVD attack.

Methods: Study subjects included 16,807 participants ( $\geq 19$ years) in the fourth Korean National Health and Nutrition Examination Surveys. From them, we selected 180 persons who had previous CVD diagnosis (angina pectoris, myocardial infarction, or stroke) and were smoking at the time of CVD diagnosis. Then, we categorized the 180 persons into two groups according to change in smoking status after the CVD: quitter and non-quitter. Logistic regression analysis was done to evaluate multivariable-adjusted association.

Results: Even after CVD diagnosis, $63.60 \%$ continued to smoke. Fully-adjusted analysis revealed that regular drinking (odds ratio $[\mathrm{OR}], 4.44)$ and presence of smokers among family members $(\mathrm{OR}, 5.86)$ were significantly $(\mathrm{P}<0.05)$ associated with greater risk of persistent smoking, whereas lower education level (OR, 0.20), larger amount of smoking (OR, 0.95), longer time since diagnosis $(\mathrm{OR}, 0.88)$, and diabetes $(\mathrm{OR}, 0.36)$ were significantly $(\mathrm{P}<0.05)$ associated with decreased risk of persistent smoking after CVD.

Conclusion: A great proportion of CVD patients tended to continue smoking in the Korean population. In order to reduce smoking rates among CVD patients further, more aggressive efforts towards smoking cessation should be continuously made with consideration of individual socioeconomic, behavioral, and clinical characteristics of CVD patients.

Keywords: Cardiovascular Diseases; Health Surveys; Korean; Risk Factors; Smoking Cessation

Received: August 28, 2012, Accepted: May 7, 2013

${ }^{*}$ Corresponding Author: Yun-Mi Song

Tel: +82-2-3410-2442, Fax: +82-2-3410-0388

E-mail: yunmisong@skku.edu

Korean Journal of Family Medicine

Copyright (C) 2013 The Korean Academy of Family Medicine

(a) This is an open-access article distributed under the terms of the Creative Commons Attribution Non-Commercial License (http://creativecommons.org/licenses/by-nc/3.0) which permits unrestricted noncommercial use, distribution, and reproduction in any medium, provided the original work is properly cited.

\section{INTRODUCTION}

Cardiovascular disease (CVD) including stroke and coronary heart disease is one of the major causes of death worldwide. ${ }^{1)}$ In 2010, CVD was the second leading cause of death in Korean people, accounting for one-fourth of entire deaths. ${ }^{2)}$ Moreover, CVD patients tend to commonly experience recurrent heart attacks or stroke even after they survive an attack of CVD. ${ }^{3)}$ For example, about 470,000 and 185,000 Americans were estimated to have recurrent coronary attacks and stroke attacks, respectively, 
each year. ${ }^{3)}$ Therefore, every possible preventive effort should be made to reduce the occurrence of primary as well as secondary CVD. A study has reported that adherence of CVD patients to recommended health behavior such as an adequate diet, regular exercise, and smoking cessation was associated with a substantially lower risk of recurrent CVD events. ${ }^{4,5)}$

Cigarette smoking is not only a well-known major modifiable risk factor for primary $\mathrm{CVD}^{6)}$ occurrence but is also associated with the outcome and prognosis of CVD. ${ }^{7-10)}$ Exposure to smoking accelerates vasomotor dysfunction, atherosclerosis, and thrombosis in multiple vascular systems. ${ }^{11)}$ The effect of smoking cessation on later mortality after the attack by coronary heart disease showed that smoking cessation resulted in a significant decrease in mortality. ${ }^{7,8)}$ Long term outcome after the attack of stroke is more frequently found to benefit from smoking cessation. ${ }^{910)}$ Despite the suggested beneficial effects of smoking cessation after CVD, the persistent smoking rate after 2 to 10 years after the CVD attack was found to be between $26 \%$ and $71 \%$ in a meta-analysis, ${ }^{8)}$ which indicates control of smoking is a very important issue in CVD survivors.

There have been studies which have evaluated the factors associated with persistent smoking after CVD. ${ }^{12-17)}$ Given that smoking patterns differ between countries, ${ }^{18)}$ factors influencing smoking habit may also differ between different populations. However, most of those studies were conducted in Western populations with only one study in the Korean population. ${ }^{17)}$ Furthermore, most studies were designed with a short-term follow-up period and findings were inconsistent between the studies. Previous studies showed that factors associated with smoking behavior after CVD attack included a history of smoking related disease, ${ }^{12,13)}$ depression, ${ }^{13,14,16)}$ Fagerstrom score, ${ }^{13,14)}$ the number of household members who smoke, ${ }^{14)}$ the number of previous attempts to quit smoking, ${ }^{15)}$ prior use of alcohol, ${ }^{16,17)}$ age when smoking began, ${ }^{16)}$ and time lapse after the CVD diagnosis. ${ }^{17)}$ In this regard, we aimed to evaluate the factors associated with persistent smoking among Korean CVD survivors using the fourth Korean National Health and Nutrition Examination Survey (KNHANES IV) data, a nationwide community survey.

\section{METHODS}

\section{Study Participants}

The study subjects were Korean men and women aged 19 years or older who participated in KNHANES IV from 2007 to 2009. The KNHANES is a cross-sectional nationwide community health survey conducted using a stratified, multistage probability sampling design and, thus, the participants in the KNHANES stand for the Korean general population. Among 24,871 participants of the KNHANES IV, we excluded 8,064 persons because of the following reasons: younger than 18 years old $(n=6,465)$; previous diagnosis of cancer $(n=504)$; and missing data about previous CVD attack $(n=5,020)$. Finally, a total of 16,807 persons were included in the present study. We defined previous CVD as having been diagnosed with angina pectoris, myocardial infarction, or stroke by doctors. Among the 16,807 study participants, 683 persons reported to have experienced CVD attack. Among the 683 persons with CVD, we identified 180 persons who were smoking at the time of CVD diagnosis. The institutional review board of the Samsung Medical Center approved the present study and acquisition of informed consent form was exempted because the data of KNHANES do not contain any identifiable personal information.

\section{Measurements}

The KNHANES survey is composed of a health interview survey, self-administered questionnaire survey, health examination, and nutrition survey. For the health interview survey, data was collected using a structured questionnaire regarding demographic characteristics, socioeconomic status, morbidities: age, sex (male or female), area of residence (rural or urban), years of education ( $\leq 9$ years or $>9$ years), occupation (economically inactive or active), marital status (married or unmarried), and previous diagnosis of diabetes, hypertension, or depression. Data on mental health included subjective stress (mild and moderate to severe) and health behaviors were collected by self-administered questionnaire. Health behaviors included smoking habit, alcohol consumption, and physical activity. Smoking habit was classified into three groups (current smoker, ex-smoker, and never smoker) based on response to questions such as "Have you ever smoked 100 cigarettes or more in your entire lifetime?" and "Do you smoke cigarettes now?" We defined 
a person as a never smoker if he/she had smoked less than 100 cigarettes through their lifetime. Ex-smoker was a person who had smoked at least 100 cigarettes through their lifetime but did not smoke at the time of KNHANES IV. Current smoker was a person who had smoked more than 100 cigarettes during entire lifetime and continued smoking until the time of the survey. Exsmoker and current smoker were combined into ever-smoker group. For ever smokers, more detailed information was collected about the age at smoking initiation, amount of smoking, duration of smoking, presence of smokers among family members, and experience of any programs that promotes smoking cessation, such as group educational program, personal counseling, advertisement, or campaign. By using the information about age at smoking initiation, duration of smoking, and age at diagnosis of cardiovascular disease, the age at quit-smoking and the time interval with regard to the age at CVD attack were estimated. We defined 'quitter' as a person who stopped smoking after the attack of CVD. We defined 'non-quitter' as a person who continued smoking even after the attack of CVD.

We defined regular drinker as a person who drank more often than once a month. Physical activity was categorized into two groups (active and inactive). If a person was involved in any one of the following types of physical activity, then he/she was categorized into the physically 'active' group: vigorous exercise (running, climbing, riding a bicycle fast, swimming fast, playing soccer, playing basketball, jump rope, playing squash, playing a single game of tennis, or weightlifting of heavy things) for more than 20 minutes at a time at a frequency of more than three times a week; moderate intensity exercise (swimming slowly, playing a double game of tennis, playing volleyball, playing badminton, playing ping-pong, or weightlifting of light thing) for more than 30 minutes at a time at a frequency of more than five times a week; and walking (including walking to work or to school) more than 30 minutes at a time at a frequency of more than five times a week.

In a health examination survey, body weight and height were measured with a person in light clothing and no shoes. Body mass index was calculated as weight in kilograms divided by height in meters squared. Then, obesity was defined based on body mass index $\left(\geq 25 \mathrm{~kg} / \mathrm{m}^{2}\right)$ according to the World Health Organization's standard for Asian people. ${ }^{19)}$ In addition, the level of glucose after a minimum 8 hours of fasting was assessed in fresh serum. Blood pressure was measured three times by trained medical staff, and the averaged value of the second and third measurement of blood pressure was used for analysis.

We identified a person with previous CVD attack based on the question asking "Have you ever been told by your doctor that you had a stroke, myocardial infarction, or angina pectoris?" We defined hypertension as follows: high systolic blood pressure $(\geq 140 \mathrm{~mm} \mathrm{Hg})$, high diastolic blood pressure $(\geq 90 \mathrm{~mm} \mathrm{Hg})$, or taking antihypertensive medication at the time of survey. We defined diabetes as follows: high fasting serum glucose level $(\geq 7$ $\mathrm{mmol} / \mathrm{L}$ ), taking oral hypoglycemic agents or insulin at the time of survey, or previous diagnosis of diabetes by a doctor.

\section{Statistical Analysis}

We compared the characteristics of the 683 persons with previous CVD with the remaining 16,124 persons without CVD to evaluate factors that are associated with CVD occurrence. As the age distribution differs markedly between the two groups, the comparison was made after standardizing for age. Among the 180 persons who were smoking at the time of CVD diagnosis, we identified 66 quit smokers and 114 persistent smokers. Then, we compared the characteristics between quitter and non-quitter in order to evaluate factors that are associated with persistent smoking after CVD attack using a chi-square test and t-test. In order to evaluate factors independently associated with persistent smoking, multi-variable adjusted logistic regression analysis was performed. Age (increase by one year), sex (male or female), duration of education ( $\leq 9$ years or $>9$ years), regular drinking (yes or no), presence of smokers among family members (yes or no), amount of smoking (number of cigarettes per day), exposure to smoking cessation program (yes or no), diabetes (yes or no), and time (increase by one year) lapse after the diagnosis of CVD were put in the model as covariates because these factors seemed to have probable association with persistent smoking in an unadjusted analysis $(\mathrm{P}<0.1)$. The statistical analyses were performed with subpopulation analysis of IBM SPSS ver. 20.0 (IBM Co., Armonk, NY, USA) with consideration of sampling weights and the complex survey design.

\section{RESULTS}

Among the 16,807 participants, 683 persons (4.06\%) 
experienced CVD attack as follows: 337 (47.60\%) experienced stroke event, 314 (47.80\%) experienced angina pectoris or myocardial infarction, and 32 (4.60\%) had both stroke and coronary heart disease. Among 683 people in the CVD group, 682 persons provided smoking history and five types of smoking habits were identified for them: never smoked $(n=360)$, stopped smoking before the attack of CVD $(\mathrm{n}=138)$, stopped smoking after CVD attack $(n=66)$, have continued smoking regardless of CVD attack ( $\mathrm{n}=114)$, newly started smoking after CVD attack $(\mathrm{n}=4)$. Mean interval between the CVD attack and KNHANES IV survey was 7.29 (standard deviation $=7.24$ ) years. Among the subjects who were smoking at the time of CVD attack, $63.30 \%$ have continued smoking.

In Table 1, characteristics between the CVD group (683 persons) and non-CVD group (16,124 persons) were compared. Mean age of the CVD group was about 20 years older than that of non-CVD group $(\mathrm{P}<0.001)$. After age standardization, we found ever-smoking was significantly more prevalent among CVD patients.

In Table 2, the characteristics of quitters and non-quitters were compared. Time interval since CVD attack was shorter for non-quitters than for quitters. Non-quitters were more likely to have achieved a higher level of education, smoke fewer cigarettes, and have smokers among family members compared with quitters. Prevalence of diabetes was lower among non-quitters than among quitters.

Table 3 shows the associations between selected variables and persistent smoking after CVD attack. Un-adjusted analysis showed that lower-level of education, amount of smoking, presence of smoking family members, time lapse since CVD attack, and diabetes mellitus were significantly associated with higher risk of persistent smoking. In the age and gender adjusted analysis, lower-level of education, amount of smoking, duration of smoking, presence of smoking family members, time since CVD attack, and diabetes mellitus were significantly associated with higher risk of persistent smoking.

Table 4 shows findings from fully-adjusted logistic regression analysis. Regular drinking and presence of smokers among family members were associated with greater risk of persistent smoking. Lower education level, larger amount of smoking, longer time since diagnosis, and diabetes were associated with decreased risk of persistent smoking after CVD.

Table 1. Age standardized characteristics* of study subjects according to the previous attack by cardiovascular disease

\begin{tabular}{|c|c|c|c|}
\hline Variable & Cardiovascular disease $(n=683)$ & No cardiovascular disease $(\mathrm{n}=16,124)$ & P-value ${ }^{\dagger}$ \\
\hline Age (y) & $66.10 \pm 0.39$ & $48.12 \pm 0.13$ & $<0.001$ \\
\hline Male & $57.05(2.3)$ & $42.61(0.4)$ & 0.04 \\
\hline Living in the rural area & $37.00(2.5)$ & $35.21(1.0)$ & 0.79 \\
\hline Duration of education less than 9 years ${ }^{\ddagger}$ & $34.74(2.2)$ & $31.01(0.6)$ & 0.57 \\
\hline Economically inactive $^{\ddagger}$ & $43.16(2.3)$ & $39.42(0.6)$ & 0.59 \\
\hline Unmarried $^{\ddagger}$ & $30.08(2.1)$ & $30.93(0.7)$ & 0.90 \\
\hline Regular drinker ${ }^{\ddagger}$ & $64.01(2.3)$ & $56.21(0.5)$ & 0.26 \\
\hline Physically inactive $^{\ddagger}$ & $39.57(2.4)$ & $43.41(0.6)$ & 0.58 \\
\hline Obese (body mass index $\geq 25 \mathrm{~kg} / \mathrm{m}^{2}$ ) & $31.71(2.2)$ & $30.02(0.5)$ & 0.80 \\
\hline Ever-smoking $^{\ddagger}$ & $60.29(2.4)$ & $38.47(0.4)$ & 0.002 \\
\hline Diabetes mellitus $^{\ddagger}$ & $9.69(2.2)$ & $6.85(0.3)$ & 0.47 \\
\hline Hypertension $^{\ddagger}$ & $34.50(2.3)$ & $23.27(0.5)$ & 0.08 \\
\hline Previously diagnosed with depression ${ }^{\ddagger}$ & $3.52(1.9)$ & $3.14(0.4)$ & 0.88 \\
\hline Moderate to severe stress, self-reported ${ }^{\ddagger}$ & $37.75(2.1)$ & $29.18(0.4)$ & 0.20 \\
\hline
\end{tabular}

Values are presented as mean \pm SD or $\%$ (SE).

*Age standardization done for all variables was done using Koran population in 2008 as a reference population. ${ }^{\dagger}$ Obtained by t-test or chisquare test. ${ }^{\ddagger}$ For some subject $(0.08 \%-11.13 \%)$, information was not available. 
Table 2. Comparison of characteristics between quitter and non-quitter among 180 persons with previous attack of cardiovascular disease

\begin{tabular}{|c|c|c|c|}
\hline Variable & Quitter $(n=66)$ & Non-quitter $(\mathrm{n}=114)$ & P-value* \\
\hline Age (y) & $64.82 \pm 1.85$ & $61.46 \pm 1.34$ & 0.15 \\
\hline Male & $89.50(3.6)$ & $80.80(3.7)$ & 0.11 \\
\hline Living in the rural area & $42.40(8.0)$ & $38.70(4.9)$ & 0.72 \\
\hline Duration of education less than 9 years & $85.10(5.0)$ & $61.10(6.2)$ & 0.01 \\
\hline Economically inactive & $59.00(7.9)$ & $55.40(5.3)$ & 0.71 \\
\hline Unmarried & $16.90^{\dagger}(5.1)$ & $21.30(4.2)$ & 0.53 \\
\hline Regular drinker & $40.70(8.3)$ & $59.60(5.4)$ & 0.07 \\
\hline Physically inactive & $41.10(7.7)$ & $52.40(6.3)$ & 0.24 \\
\hline Obese (body mass index $\geq 25 \mathrm{~kg} / \mathrm{m}^{2}$ ) & $34.90^{\dagger}(8.0)$ & $28.10^{\dagger}(4.6)$ & 0.46 \\
\hline Age at starting smoking (y) & $21.43 \pm 0.74$ & $23.17 \pm 0.92$ & 0.17 \\
\hline Amount of smoking (cigarettes per day) & $18.18 \pm 1.64$ & $14.50 \pm 0.78^{\ddagger}$ & 0.03 \\
\hline Duration of smoking (y) & $38.25 \pm 1.96$ & $38.30 \pm 1.59$ & 0.99 \\
\hline Smokers among family members & $11.40(4.1)$ & $39.50(5.9)$ & $<0.001$ \\
\hline Exposure to smoking cessation program & $76.00(6.8)$ & $88.00^{\dagger}(3.3)$ & 0.08 \\
\hline Categories of cardiovascular disease & & & 0.28 \\
\hline Stroke and coronary heart disease & $4.20(2.2)$ & $2.20(1.1)$ & \\
\hline Stroke & $56.20(8.4)$ & $44.90(5.1)$ & \\
\hline Coronary heart disease & $39.60(8.3)$ & $52.90(5.2)$ & \\
\hline Time lapse after the diagnosis of cardiovascular disease (y) & $9.95 \pm 1.00$ & $6.02 \pm 0.57^{\ddagger}$ & 0.01 \\
\hline Diabetes mellitus & $39.50^{\dagger}(9.7)$ & $18.40^{\dagger}(4.2)$ & 0.02 \\
\hline Hypertension & $64.40(7.5)$ & $54.20^{\dagger}(5.4)$ & 0.26 \\
\hline Previously diagnosed with depression & $4.50(1.8)$ & $5.30(2.3)$ & 0.78 \\
\hline Moderate to severe stress, self-reported & $18.10(5.1)$ & $31.40(5.4)$ & 0.11 \\
\hline
\end{tabular}

Values are presented as mean $\pm \mathrm{SD}$ or $\%$ (SE).

* Obtained by t-test or chi-square test. ${ }^{\dagger}$ For some subjects $(0.88 \%-15.15 \%)$, information was not available. ${ }^{\ddagger}$ Imputation for missing data (for two subjects) was done with mean value of subjects in his/her age group.

\section{DISCUSSION}

To our knowledge, this is the first study to evaluate factors associated with persistent smoking even after the diagnosis of CVD in nationally representative samples from the Korean population. Our study reveals that CVD patients who drink alcohol regularly or have smoking family members at home were less likely to quit smoking. Low educational level, greater amount of smoking, longer interval after the diagnosis of CVD, and having diabetes mellitus were factors associated with decreased persistent smoking.
A previous study showed that the risk of persistent smoking was about 1.5 times higher among coronary heart disease patients than stroke patients. ${ }^{16)}$ It was presumed that the labeling effect of stroke was stronger than that of coronary heart disease, given the greater undesirable consequences of stroke including neurological impairment. However, there was no significant difference between stroke patients and coronary heart disease patients in the present study.

Experiencing a smoking-related disease such as CVD might be a potential trigger factor for smoking cessation. ${ }^{12)}$ However, there have been several studies that do not support this assumption. A 
Table 3. Factors associated with persistent smoking after the diagnosis of cardiovascular disease

\begin{tabular}{|c|c|c|c|c|}
\hline \multirow{2}{*}{ Variable } & \multicolumn{2}{|c|}{ Unadjusted model } & \multicolumn{2}{|c|}{ Age and gender adjusted model } \\
\hline & OR & $95 \%$ CI & OR & $95 \%$ CI \\
\hline Age (increase by one year) & 0.98 & $(0.94-1.01)$ & 0.97 & $(0.94-1.01)$ \\
\hline Male & 0.49 & $(0.21-1.18)$ & 0.42 & $(0.16-1.09)$ \\
\hline Living in the rural area & 0.87 & $(0.38-1.95)$ & 0.93 & $(0.42-2.08)$ \\
\hline Duration of education less than 9 years & 0.28 & $(0.11-0.70)$ & 0.26 & $(0.10-0.71)$ \\
\hline Economically inactive & 0.86 & $(0.39-1.90)$ & 1.12 & $(0.46-2.73)$ \\
\hline Unmarried & 1.33 & $(0.55-3.24)$ & 1.01 & $(0.36-2.85)$ \\
\hline Regular drinker & 2.16 & $(0.92-5.03)$ & 2.20 & $(0.98-4.99)$ \\
\hline Physically inactive & 1.57 & $(0.73-3.38)$ & 1.58 & $(0.70-3.58)$ \\
\hline Obese $\left(25 \mathrm{~kg} / \mathrm{m}^{2} \leq\right.$ body mass index $)$ & 0.76 & $(0.32-1.77)$ & 0.78 & $(0.33-1.81)$ \\
\hline Age at starting smoking (increase by one year) & 1.02 & $(0.99-1.06)$ & 1.01 & $(0.97-1.05)$ \\
\hline Amount of smoking (cigarettes per day) & 0.96 & $(0.93-0.99)$ & 0.96 & $(0.93-0.99)$ \\
\hline Duration of smoking (increase by one year) & 1.00 & $(0.97-1.03)$ & 1.12 & $(1.02-1.23)$ \\
\hline Smokers among family members & 5.07 & $(2.05-12.53)$ & 4.62 & $(1.89-11.28)$ \\
\hline No exposure to smoking cessation program & 0.43 & $(0.17-1.12)$ & 0.42 & $(0.14-1.24)$ \\
\hline \multicolumn{5}{|l|}{ Final diagnosis } \\
\hline Stroke and coronary heart disease & 1 & Reference & 1 & Reference \\
\hline Stroke & 1.52 & $(0.32-7.15)$ & 1.49 & $(0.30-7.52)$ \\
\hline Coronary heart disease & 2.55 & $(0.51-12.69)$ & 2.21 & $(0.42-11.80)$ \\
\hline $\begin{array}{l}\text { Time lapse after the diagnosis of cardiovascular disease } \\
\text { (increase by one year) }\end{array}$ & 0.92 & $(0.87-0.97)$ & 0.92 & $(0.86-0.97)$ \\
\hline Diabetes mellitus & 0.35 & $(0.14-0.88)$ & 0.32 & $(0.13-0.83)$ \\
\hline Hypertension & 0.65 & $(0.31-1.39)$ & 0.74 & $(0.34-1.59)$ \\
\hline Previously diagnosed with depression & 1.19 & $(0.35-4.10)$ & 1.19 & $(0.39-3.65)$ \\
\hline Moderate to severe stress, self-reported & 2.07 & $(0.84-5.10)$ & 1.89 & $(0.75-4.77)$ \\
\hline
\end{tabular}

OR: odds ratio, CI: confidence interval.

study in North East Melbourne reported that $63.00 \%$ of stroke survivors had continued smoking until 5 years after their stroke attack. $^{20)}$ The smoking rate of coronary heart disease patients was reported to be $60.40 \%$ in a study conducted in two university affiliated hospitals located in Seoul. ${ }^{17)}$ Our study also revealed that a high proportion (63.3\%) of CVD patients continued smoking even after diagnosis of CVD. These findings suggest that experience with smoking-related diseases is not always enough to induce smoking cessation. Therefore, more aggressive efforts should be put towards smoking cessation for CVD patients who smoke.
Alcohol drinking was found to be associated with significantly higher risk of continued smoking after CVD. Other studies in CVD patients also showed a positive association between cigarette smoking and alcohol consumption. ${ }^{16,17,21)}$ These findings suggest that not only the smoking but also alcohol drinking should be the target of behavioral changes for CVD patients.

In a previous study, the number of household members who smoke predicted smoking status of stroke survivors. ${ }^{14)}$ Although we could not evaluate the association between the number of smokers among family members and persistent smoking due to the lack of available data, presence of smokers among family 
Table 4. Multivariable-adjusted associations between the probable risk factors and persistent smoking after the diagnosis of cardiovascular disease

\begin{tabular}{lcc}
\hline \multicolumn{1}{c}{ Variable } & \multicolumn{2}{c}{ Multivariable adjusted model* } \\
\cline { 2 - 3 } & Odds ratio & 95\% confidence interval \\
\hline Age (increase by one year) & 1.01 & $(0.96-1.07)$ \\
Male & 0.30 & $(0.06-1.67)$ \\
Duration of education less than 9 years & 0.20 & $(0.06-0.61)$ \\
Regular drinker & 4.44 & $(1.45-13.59)$ \\
Amount of smoking (cigarettes per day) & 0.95 & $(2.03-0.99)$ \\
Smokers among family members & 5.86 & $(0.20-3.18)$ \\
No exposure to smoking cessation program & 0.79 & $(0.80-0.96)$ \\
Time lapse after the diagnosis of cardiovascular disease (increase by one year) & 0.88 & $(0.13-0.98)$ \\
Diabetes mellitus & 0.36 & \\
\hline
\end{tabular}

*Estimated by logistic regression analysis.

members was a risk factor for persistent smoking among CVD patients in our study. Therefore, familial support should be provided for smoking cessation of CVD patients.

Generally, light smokers are more likely to quit smoking due to lower nicotine dependency than heavy smokers among the general population. ${ }^{22)}$ However, we found that quit-smoking was positively associated with the amount of smoking. Similar findings were observed in a previous study ${ }^{12)}$ and the authors suggested that smokers with higher cigarette consumption are more likely to recognize the causal relationship between smoking and their diseases, and thus are more strongly motivated to quit smoking. ${ }^{12)}$ Therefore, special attention should be paid to CVD patients who smoke fewer cigarettes.

Interestingly, a low level of education was a significant predictor of smoking cessation in our study. Regarding this issue, previous studies have shown contradictory findings. A higher educated person is more likely to be a successful quit-smoker among the general population because they are more healthconscious. ${ }^{23)}$ The recently raised price of cigarettes may be related with the findings of our study. Although education level does not fully represent income level, smokers who achieved a lower level of education would be more sensitive to the raised price of cigarettes. However, we could not investigate this issue.

We found that time lapse after the diagnosis of CVD was positively associated with smoking cessation. This finding is inconsistent with the findings from studies in western populations in which many CVD patients quit smoking immediately after the diagnosis of CVD and then gradually restarted smoking with time lapse. ${ }^{12,24)}$ This difference between ours and other studies may be related to the difference in time of introduction of a smoking restriction policy between Korea and western countries where smoking restriction policies were started much earlier. ${ }^{25)}$ In Korea, smoking cessation rate is also gradually increasing among the general population with the strengthening of anti-smoking policy over the last decade, ${ }^{26)}$ which may be reflected in our study.

Our study has both limitations and strengths. Information bias could be possible regarding CVD history and smoking habit because the information was obtained by questionnaire instead of medical record review or laboratory testing such as a cotinine level test. Our study was conducted in a cross-sectional design and a cause-effect relation may be uncertain especially for the changeable factors such as alcohol drinking. However, the finding from our study could be more generalizable to the Korean population because we used KNHANES data for which nationwide probability sampling was applied. In addition, we evaluated the factors associated with changes in smoking behavior over a long time period ( 7.29 years).

In conclusion, this study in Korean representative samples shows that a materially great proportion of CVD patients tended to continue smoking. Drinking alcohol regularly and presence of smokers among family members were associated with an increased risk of persistent smoking, while low educational level, 
greater amount of smoking, longer interval after the diagnosis of CVD, and having diabetes mellitus were the factors associated with decreased risk of persistent smoking. These findings suggest that more aggressive efforts towards smoking cessation should be made with careful consideration of individual socioeconomic, behavioral, and clinical characteristics of CVD patients.

\section{CONFLICT OF INTEREST}

No potential conflict of interest relevant to this article was reported.

\section{ACKNOWLEDGMENTS}

This research was supported by the Basic Science Research Program through the National Research Foundation of Korea funded by the Ministry of Education, Science and Technology (2011-0013545 and 2010-0029113).

\section{REFERENCES}

1. Tu JV, Nardi L, Fang J, Liu J, Khalid L, Johansen H, et al. National trends in rates of death and hospital admissions related to acute myocardial infarction, heart failure and stroke, 1994-2004. CMAJ 2009; 180:E118-25.

2. Statistics Korea. Statistics Korea: 2010 cause of death statistics in Korean [Internet]. Daejeon: Statistics Korea; c2010 [cited 2011 Sep 8]. Available from: http://kostat.go.kr.

3. Roger VL, Go AS, Lloyd-Jones DM, Benjamin EJ, Berry JD, Borden WB, et al. Heart disease and stroke statistics--2012 update: a report from the American Heart Association. Circulation 2012;125:e2-e220.

4. Chow CK, Jolly S, Rao-Melacini P, Fox KA, Anand SS, Yusuf S. Association of diet, exercise, and smoking modification with risk of early cardiovascular events after acute coronary syndromes. Circulation 2010;121:750-8.

5. Towfighi A, Markovic D, Ovbiagele B. Impact of a healthy lifestyle on all-cause and cardiovascular mortality after stroke in the USA. J Neurol Neurosurg Psychiatry 2012;83:146-51.
6. Burns DM. Epidemiology of smoking-induced cardiovascular disease. Prog Cardiovasc Dis 2003;46:11-29.

7. Hasdai D, Garratt KN, Grill DE, Lerman A, Holmes DR Jr. Effect of smoking status on the long-term outcome after successful percutaneous coronary revascularization. $\mathrm{N}$ Engl J Med 1997;336:755-61.

8. Wilson K, Gibson N, Willan A, Cook D. Effect of smoking cessation on mortality after myocardial infarction: metaanalysis of cohort studies. Arch Intern Med 2000;160:93944.

9. Myint PK, Welch AA, Bingham SA, Luben RN, Wareham NJ, Day NE, et al. Smoking predicts long-term mortality in stroke: The European Prospective Investigation into Cancer (EPIC)-Norfolk prospective population study. Prev Med 2006;42:128-31.

10. Kammersgaard LP. Survival after stroke: risk factors and determinants in the Copenhagen Stroke Study. Dan Med Bull 2010;57:B4189.

11. Ambrose JA, Barua RS. The pathophysiology of cigarette smoking and cardiovascular disease: an update. J Am Coll Cardiol 2004;43:1731-7.

12. Twardella D, Loew M, Rothenbacher D, Stegmaier C, Ziegler $\mathrm{H}$, Brenner $\mathrm{H}$. The diagnosis of a smoking-related disease is a prominent trigger for smoking cessation in a retrospective cohort study. J Clin Epidemiol 2006;59:82-9.

13. Vogiatzis I, Tsikrika E, Sachpekidis V, Pittas S, Kotsani A. Factors affecting smoking resumption after acute coronary syndromes. Hellenic J Cardiol 2010;51:294-300.

14. Sienkiewicz-Jarosz H, Zatorski P, Baranowska A, Ryglewicz D, Bienkowski P. Predictors of smoking abstinence after firstever ischemic stroke: a 3-month follow-up. Stroke 2009;40: 2592-3.

15. Rigotti NA, McKool KM, Shiffman S. Predictors of smoking cessation after coronary artery bypass graft surgery: results of a randomized trial with 5-year follow-up. Ann Intern Med 1994;120:287-93.

16. Ballard J, Kreiter KT, Claassen J, Kowalski RG, Connolly ES, Mayer SA. Risk factors for continued cigarette use after subarachnoid hemorrhage. Stroke 2003;34:1859-63.

17. Kim HM, Choi CJ, Seung KB, Chung WS, Kahng HB. Smoking behavior following diagnosis of the coronary artery disease and factors influencing smoking cessation. J Korean 
Acad Fam Med 2005;26:629-35.

18. Degenhardt L, Chiu WT, Sampson N, Kessler RC, Anthony JC, Angermeyer M, et al. Toward a global view of alcohol, tobacco, cannabis, and cocaine use: findings from the $\mathrm{WHO}$ World Mental Health Surveys. PLoS Med 2008;5:e141.

19. Weisell RC. Body mass index as an indicator of obesity. Asia Pac J Clin Nutr 2002;11 Suppl 8:S681-4.

20. Gall SL, Dewey HM, Thrift AG. Smoking cessation at 5 years after stroke in the North East Melbourne stroke incidence study. Neuroepidemiology 2009;32:196-200.

21. Nakamura Y, Sakata K, Yanagawa H. Relationships between smoking habits and other behavior factors among males: from the results of the 1990 National Cardiovascular Survey in Japan. J Epidemiol 1996;6:87-91.

22. Myung SK, Seo HG, Park S, Kim Y, Kim DJ, Lee DH, et al. Sociodemographic and smoking behavioral predictors associated with smoking cessation according to follow-up periods: a randomized, double-blind, placebo-controlled trial of transdermal nicotine patches. J Korean Med Sci 2007;22:
1065-70.

23. Hyland A, Li Q, Bauer JE, Giovino GA, Steger C, Cummings KM. Predictors of cessation in a cohort of current and former smokers followed over 13 years. Nicotine Tob Res 2004;6 Suppl 3:S363-9.

24. Ives SP, Heuschmann PU, Wolfe CD, Redfern J. Patterns of smoking cessation in the first 3 years after stroke: the South London Stroke Register. Eur J Cardiovasc Prev Rehabil 2008; 15:329-35.

25. Fagerstrom K, Nakamura M, Cho HJ, Tsai ST, Wang C, Davies $S$, et al. Varenicline treatment for smoking cessation in Asian populations: a pooled analysis of placebo-controlled trials conducted in six Asian countries. Curr Med Res Opin 2010;26:2165-73.

26. Park EJ, Koh HK, Kwon JW, Suh MK, Kim H, Cho SI. Secular trends in adult male smoking from 1992 to 2006 in South Korea: age-specific changes with evolving tobaccocontrol policies. Public Health 2009; 123:657-64. 